\title{
Repurposing Drugs in Oncology (ReDO)—mebendazole as an anti-cancer agent
}

\author{
Pan Pantziarka1,2, Gauthier Bouche ${ }^{1}$, Lydie Meheus ${ }^{1}$, Vidula Sukhatme ${ }^{3}$ and Vikas P. Sukhatme ${ }^{3,4}$ \\ ${ }^{1}$ Anticancer Fund, Brussels, 1853 Strombeek-Bever, Belgium \\ ${ }^{2}$ The George Pantziarka TP53 Trust, London KT1 2JP, UK \\ ${ }^{3}$ GlobalCures, Inc, Newton, MA 02459, USA \\ ${ }^{4}$ Beth Israel Deaconess Medical Centre and Harvard Medical School, Boston, MA 02215, USA
}

Correspondence to: Pan Pantziarka. Email: anticancer.org.uk@gmail.com

\begin{abstract}
Mebendazole, a well-known anti-helminthic drug in wide clinical use, has anti-cancer properties that have been elucidated in a broad range of pre-clinical studies across a number of different cancer types. Significantly, there are also two case reports of anti-cancer activity in humans. The data are summarised and discussed in relation to suggested mechanisms of action. Based on the evidence presented, it is proposed that mebendazole would synergise with a range of other drugs, including existing chemotherapeutics, and that further exploration of the potential of mebendazole as an anti-cancer therapeutic is warranted. A number of possible combinations with other drugs are discussed in the Appendix.
\end{abstract}

Keywords: drug repurposing, anti-helminthic, metronomic chemotherapy, cancer, ReDO Project

Published: $10 / 07 / 2014$

Received: $24 / 03 / 2014$

ecancer 2014, 8:443 DOI: 10.3332/ecancer.2014.443

Copyright: (c) the authors; licensee ecancermedicalscience. This is an Open Access article distributed under the terms of the Creative Commons Attribution License (http://creativecommons.org/licenses/by/3.0), which permits unrestricted use, distribution, and reproduction in any medium, provided the original work is properly cited. 


\section{Introduction}

Mebendazole (MBZ) is a broad-spectrum benzimidazole anti-helminthic drug, in the same class as albendazole, flubendazole, oxfendazole, and others. It is commonly prescribed to treat a range of parasitical worm infections, including threadworm, tapeworms, roundworms, and other nematode and trematode infections in humans and domestic animals. MBZ is available as a generic drug; common trade names have included Vermox (Janssen Pharamceutica) and Ovex (McNeil Products Ltd) in the US and Europe. It is generally available over the counter in European countries, but the last US manufacturer, Teva Pharmaceuticals, ceased production at the end of 2011, although the drug retains US Food and Drug Administration (FDA) approval. It is available in the US from compounding pharmacies, for example, Pavillion Compounding Pharmacy in Atlanta.

\section{Dosage}

For human use, the most common formulation of MBZ is as $100 \mathrm{mg}$ chewable tablets. The dosage varies according to the type of helminthic infection being treated. Pinworms are treated with a single $100 \mathrm{mg}$ treatment, whereas roundworms or hookworms are treated with $100 \mathrm{mg}$ twice a day for three days. MBZ, along with albendazole, is also used on a long-term basis for the treatment of human cystic and alveolar echinococcosis (also known as hydatid disease). According to the guidelines published by the World Health Organisation (http://whqlibdoc.who.int/bulletin/1996/Vol74-No3/bulletin_1996 74\%283\%29 231-242.pdf), long-term treatment of cystic echinococcosis using MBZ is at a dosage of $40-50 \mathrm{mg} / \mathrm{kg} / \mathrm{day}$ for at least 3-6 months. For alveolar echinococcosis, the dose is $40-50 \mathrm{mg} / \mathrm{kg} / \mathrm{day}$, with treatment for at least two years, and possibly longer for patients with inoperable disease. Indeed, there are documented cases of treatment periods of ten or more years $[1,2]$.

\section{Toxicity}

MBZ has low toxicity, though patients may suffer from transient symptoms, such as abdominal pain and diarrhoea in cases of massive infection and excretion of parasites. Hypersensitivity reactions, such as rash, urticaria, and angioedema, have been observed on rare occasions. MBZ is contraindicated during pregnancy. Caution is also recommended in treating infants below the age of 2 , primarily due to a lack of data in such cases [3].

In the case of long-term administration of MBZ for echinococcosis, the evidence is that, in general, the treatment is well tolerated, but the specific treatment for some patients has to be discontinued. For example, in one open-labelled observational study, the patients treated with MBZ for alveolar echinococcosis (average: 24 months) experienced few adverse reactions, and in only three patients (of 17), the treatment was changed to albendazole due to intolerable side effects (reversible alopecia, psychological disturbance, and drop in performance) [4].

\section{Pharmacokinetics}

First-pass metabolism of MBZ ensures that only about $20 \%$ of the oral dose reaches systemic circulation, with maximum plasma concentration reached 2-4 hours post-administration. Dosing with a high-fat meal is known to modestly increase bioavailability [5]. Chronic dosing of MBZ increases plasma concentration by a factor of between two and three compared to single dose [3, 6]. In one series of patients treated with chronic MBZ at a dose of $40 \mathrm{mg} / \mathrm{kg} / \mathrm{day}$ for hydatid disease, the mean peak plasma level was $137.4 \mathrm{ng} / \mathrm{ml}$ $[0.47 \mu \mathrm{M}]$ after a single dose of $10 \mathrm{mg} / \mathrm{kg}$; however, there was high inter-patient variability $(99.4-500 \mathrm{ng} / \mathrm{ml}[0.34-1.69 \mu \mathrm{M}])$. For patients not on chronic treatment, an initial treatment of MBZ at the same dose produced a mean peak plasma level of $69.5 \mathrm{ng} / \mathrm{ml}[0.24 \mu \mathrm{M}]$, $(17.5-116.2 \mathrm{ng} / \mathrm{ml}[0.06-0.39 \mu \mathrm{M}])[6]$.

The poor bioavailability has long been recognised, and strategies to improve this remain actively researched, these strategies have included alternative formulations with vegetable oils [7-9], altering the crystalline structure of MBZ [10] and investigations into PEGylation [11]. 
Albendazole and MBZ interact with cimetidine, which inhibits metabolism and has been documented to increase MBZ plasma levels, (maximum serum levels rose to $82.3 \pm 41.8 \mathrm{ng} / \mathrm{ml}[0.28 \pm 0.14 \mu \mathrm{M}]$ from $55.7 \pm 30.2 \mathrm{ng} / \mathrm{ml}[0.19 \pm 0.10 \mu \mathrm{M}]$, on $1.5 \mathrm{~g}$ of MBZ following chronic dosing of cimetidine at $400 \mathrm{mg}$ three times a day for 30 days) [12]. This may be an important interaction with clinical relevance in that it suggests a strategy to increase bioavailability should that be required to increase the anti-cancer effect. Given that cimetidine may also have some anti-cancer activity [13], it also suggests that an investigation into possible synergies with MBZ over and above the effect on bioavailability would be an interesting avenue to explore.

High intra- and inter-patient variability may be an important factor in assessing response to MBZ as a possible anti-cancer therapeutic. However, it is clear that plasma levels achieved by chronic and high-dosing schedules are in the range necessary for clinical activity based on the pre-clinical evidence assessed in the following section.

\section{Pre-clinical evidence in cancer-in vitro and in vivo}

In 2002, Mukhopadhyay and colleagues showed that MBZ induced a dose- and time-dependant apoptotic response in a range of lung cancer cell lines [14, 15], with an IC50 of $\sim 0.16 \mu \mathrm{M}$. Cells were arrested in the G2-M phase before undergoing apoptosis. Just as importantly, MBZ had no effect on normal HUVECs or WI38 fibroblasts, even at a concentration of $1 \mu \mathrm{M}$. Overall, the in vitro results showed that MBZ inhibited lung cancer cell growth 5-fold compared to controls. Additionally, the authors confirmed the growth inhibitory effects of MBZ against breast, ovary, colon carcinomas, and osteosarcoma, producing IC50s that varied from 0.1 to $0.8 \mu \mathrm{M}$.

To test the in vivo response to the MBZ treatment, nu/nu mice were inoculated with subcutaneous injections of $\mathrm{H} 460$ non-small cell lung cancer cells [14]. Animals with established tumours ( $\sim 3 \mathrm{~mm}$ diameter) were treated with $1 \mathrm{mg}$ orally of MBZ every other day. Treated animals showed a dose-dependent arrest in tumour growth. The experiment was also repeated with $\mathrm{C} 3 \mathrm{H}$ mice and the K1735 mouse cell line, and MBZ inhibited tumour growth in this syngeneic mouse model also. Mice treated with MBZ showed no side effects. Finally, the investigators also assessed whether MBZ might inhibit the formation of lung metastases and injected A549 cells into the tail vein of mice. In untreated controls, approximately 300 metastatic colonies appeared in the lungs by 21 days. Mice treated with $1 \mathrm{mg}$ of MBZ every other day showed a mean colony count $80 \%$ lower than controls. Treatment with the established anti-microtubule agent paclitaxel showed no such reduction in colony formation.

Further pre-clinical evidence of MBZ anti-cancer activity was shown in adrenocortical cancer in 2008 [16], both in vitro and in vivo. H295R, SW-13 and WI-38 (normal fibroblast) cells lines were treated with different concentrations of MBZ in vitro, and the two cancer cells lines showed dose-dependent growth arrest, with IC50 of $0.23 \mu \mathrm{M}$ for H295R and $0.27 \mu \mathrm{M}$ for SW-13 cells, with no effect on the normal fibroblast cells. Tumour spheroid inhibition was tested against a dose of $1 \mu \mathrm{M}$ of MBZ, which completely disaggregated the tumour spheroids and killed all cancer cells in about 20 days.

In vivo treatment of athymic nude mouse models of adrenocortical cancer showed that treatment with $1 \mathrm{mg}$ and $2 \mathrm{mg}$ MBZ significantly inhibited tumour growth in both implanted adrenocortical cancers. While there was little difference between the response of the primary tumours to $1 \mathrm{mg}$ and $2 \mathrm{mg}$ doses, the latter dose inhibited the formation of metastases from $50 \%$ of controls to $75 \%$. No side effects were noted in the treated animals. Of note, a dose of $1 \mathrm{mg} /$ day in a mouse weighing $20 \mathrm{gm}$ corresponds to a human dose of approximately $500 \mathrm{mg}$ daily for a $70 \mathrm{~kg}$ person, if extrapolated by surface area.

In 2008, the in vitro activity of MBZ against chemoresistant melanoma cell lines was assessed by Doudican et al [17]. A screening of 2000 small molecules against melanoma cells lines picked out ten compounds that had inhibitory action against the M-14 and SK-Mel-19 chemoresistant melanoma cells lines, but were non-toxic to normal melanocytes. Of these ten compounds, four were benzimidazoles — mebendazole, albendazole, fenbendazole, and oxybendazole — and of these four compounds, MBZ was selected for more detailed analysis based on its relative lack of toxicity and well-characterised pharmacokinetics. MBZ was shown to induce dose-dependent apoptosis in both cell lines with an average IC50 of $0.32 \mu \mathrm{M}$, while the equivalent for the non-cancerous melanocyte cell line was IC50 of $1.9 \mu \mathrm{M}$. MBZ also had the greatest inhibitory effect against the melanoma cells of the four benzimidazoles tested. 
Subsequently, MBZ was shown to inhibit human melanoma xenograft growth in athymic female nude mice fed $1 \mathrm{mg}$ or $2 \mathrm{mg}$ oral MBZ every other day [18]. Tumour growth was reduced by $83 \%$ for the $1 \mathrm{mg}$ dose and $77 \%$ for the $2 \mathrm{mg}$ in comparison to controls. This was comparable to the growth inhibitory activity of $100 \mathrm{mg} / \mathrm{kg}$ of temozolomide (TMZ) by an intraperitoneal injection for 5 days, used as a positive control as it represents a well-characterised option for melanoma treatment. These results showed that oral MBZ produced equivalent responses to high-dose TMZ, but with no observed side effects.

MBZ activity in glioblastoma multiforme (GBM) was discovered serendipitously in 2011 by investigators, who observed that GBM xenografts were failing after mice models were fed albendazole to fight a spate of pin worm infections [19]. Further investigation showed that both albendazole- and MBZ-induced apoptosis in two GBM cells lines in vitro and in vivo. The in vitro IC50 of MBZ was $0.24 \mu \mathrm{M}$ in the GL261 mouse glioma line, and $0.1 \mu \mathrm{M}$ in the 060919 human GBM. In vivo results showed that oral MBZ treatment significantly extended mean survival up to $63 \%$ in syngeneic and xenograft orthotopic mouse glioma models.

Screening of compounds for activity against colon cancer cell lines also identified MBZ as a candidate molecule in work by Nygren and colleagues [20]. The authors set out to screen 1600 existing drugs for activity against two well-established colon cancer cell lines (HCT 116 and RKO) and found 64 candidate drugs, including a cluster of benzimidazoles (albendazole, mebendazole, oxybendazole and fenbendazole). Of these, further analysis was performed on MBZ and albendazole because, in the words of the authors, 'they are registered pharmaceuticals for clinical use in humans, thus easily accessible for clinical testing'.

Diagnosis-specific activity was assessed using the $\mathrm{NCl} 60 \mathrm{z}$ score data, which showed a high level of activity against leukaemia, colon cancer, CNS and melanoma panels of cell lines, with lesser activity in breast, ovarian, renal and NSCLC lines. It should be noted that the leukaemia panel had the highest level of sensitivity to MBZ, a finding that has not been further investigated to date. In the colon cancer panel, $80 \%$ of cells lines were sensitive to MBZ. Detailed in vitro treatment against five colon cancer cell lines (HCT 116, RKO, HT29, HT-8 and SW626), showed that all displayed IC50 of $\angle 5 \mu \mathrm{M}$, whereas the drug was largely inactive in the non-malignant cell lines.

Some work on in vitro efficacy against a chemoresistant breast cancer cell line (SKBr-3) was performed by Coyne and colleagues in 2013 [21]. A range of benzimidazoles, including MBZ and albendazole, were tested and found to cause significant growth arrest and apoptosis, with flubendazole and MBZ showing the greatest level of cytotoxic activity. MBZ reduced cell survival by $63.1 \%$ at a dose of $0.5 \mu \mathrm{M}$.

Finally, Schmit showed that a range of benzimidazoles, including MBZ, possess anti-neoplastic activity against the DS 17 canine osteosarcoma cell line in vitro [22]. Canine osteosarcoma is an excellent animal model of the human disease. The results obtained showed that MBZ induced cell cycle arrest and apoptosis at MBZ doses that are clinically achievable with oral dosing.

\section{Human data in cancer}

No clinical trials of MBZ as a cancer treatment have been completed to date. However, there are two well-documented case reports in the literature in favour of re-purposing MBZ as an anti-cancer therapy.

In 2011, a case of long-term tumour control in metastatic adrenocortical cancer was published [23]. Adrenocortical cancer is a relatively rare malignancy with few treatment options in the case of non-resectable disease. The patient had experienced disease progression despite multiple chemotherapeutic protocols and several rounds of surgery. After all other treatment options had been exhausted, the patient discovered the pre-clinical evidence of MBZ action against adrenocortical cancer via Pubmed and forwarded the information to the clinicians, who agreed to use it based on this evidence and the relatively low toxicity of treatment. Monotherapy commenced with MBZ at a typical anti-helminthic dose of $100 \mathrm{mg}$ twice a day. The patient experienced some regression in metastatic lesions, and overall the disease remained stable for 19 months of MBZ monotherapy, tolerating the treatment without side effects, and his quality of life returned to his baseline prior to his initial surgery. However, 24 months after the commencement of oral MBZ a scan showed disease progression, and everolimus was added to the MBZ but without additional benefit in disease control.

A case of metastatic colon cancer treated with MBZ was described by Peter Nygren and Rolf Larsson in 2013 [24]. Here, a 74-year-old patient suffering from progressive metastatic colon cancer had been treated first with capecitabine, oxaliplatin, and bevacizumab, and then 
by capecitabine and irinotecan in the face of disease progression, and who had no standard treatment options available was started on an oral dose of MBZ of $100 \mathrm{mg}$ twice a day. MBZ was selected based on the author's previous pre-clinical work with MBZ [20]. After six weeks of monotherapy, radiological evaluation showed near complete remission of metastatic lesions in the lungs and lymph nodes and a good partial remission in the liver. However, the patient experienced elevated liver enzymes (AST and ALT), so MBZ was temporarily stopped and then started at half the dose, with the patient reporting no ill effects. Liver enzymes normalised and a subsequent round of CT scans confirmed the initial disease response. After ceasing treatment for approximately three months, the patient developed brain metastases that were treated with radiotherapy, following by evidence of disease in the lymph nodes. MBZ treatment was not recommenced following the discovery of the brain metastases or in subsequent disease progression. A further five patients have been treated, with one experiencing a minor remission [Private communication from Peter Nygren].

\section{Clinical trials}

There are currently two clinical trials of MBZ in cancer, both for brain tumours.

One is a Phase I open label study, at John Hopkins Hospital, of MBZ in newly diagnosed high-grade glioma patients receiving temozolomide (http://clinicaltrials.gov/ct2/show/NCT01729260). Patients recruited to the trial are treated on a 28 day cycle of $500 \mathrm{mg}$ MBZ tablets three times a day. The primary end point is to determine the maximum tolerated dose of MBZ with temozolomide. A secondary end point is to determine if MBZ with current standard of care can slow tumour progression. Study completion is scheduled for November 2014 (at the time of writing in January 2014).

The other clinical trial is at Cohen Children's Medical Centre of New York in paediatric patients with low-grade gliomas (http://clinicaltrials. gov/ct2/show/NCT01837862). This is a Phase I and II pilot study of MBZ in combination with vincristine, carboplatin, and temozolomide. The study design is non-randomised and open label, with comparison between standard of care and standard of care plus MBZ arms. The MBZ dose is $100 \mathrm{mg}$ twice a day over the 70 weeks of treatment. The primary objective of the Phase I part of the trial is to determine if the standard dose of MBZ $100 \mathrm{mg}$ twice daily is 'well-tolerated' when used in combination with the current three-drug regimen. At the end of Phase I potential subjects will be offered the chance to receive the three-drug regimen + MBZ for 70 weeks, or else to enrol as part of the control group receiving the three-drug regimen alone. For the Phase II portion of the study, the outcome variables of interest are progression-free and overall survival. Study completion is scheduled for December 2017 (as of December 2013).

\section{Mechanism of action}

The anti-parasitic action of MBZ is due to its action as a microtubule-disrupting agent acting to prevent the polymerisation of tubulin in the gut of helminths, causing the parasites to die [25]. Tubulin is vital to cell division and is therefore a cancer target for several widely used chemotherapy drugs, including paclitaxel, colchicine, and vincristine. MBZ, as with the other benzimidazoles, binds to the colchicine-binding domain of tubulin [26].The inhibition of tubulin polymerisation by MBZ has been confirmed in vitro in a glioblastoma model [19] and in a melanoma model [17]. The latter work suggested that the apoptotic response to microtubule disruption is mediated by Bcl-2 phosphorylation. Subsequent work on melanoma confirmed this result, and also showed that MBZ decreased the levels of X-linked inhibitor of apoptosis (XIAP) [18], but to date this has not been confirmed in non-melanoma cell lines.

While there are rare reports of reversible alopecia, urticaria, rash, gastro-intestinal upset, leukopenia, and neutropenia in some patients treated with high-dose MBZ, all adverse effects associated with other microtubule disruption agents, there do not appear to be any reports of peripheral neuropathy, which is commonly considered a classic adverse effect of microtubule disrupting agents, including the taxanes and the vinca alkaloids [27]. While this may suggest that the action of MBZ is independent of microtubule disruption, it may also be related to the fact that MBZ acts via the colchicine-binding domain, and that like colchicine, there is little effect in terms of neuropathic pain [28]. Of course, it is also possible that the anticancer activity of MBZ is mediated by additional molecular targets yet to be elucidated. 
MBZ appears to be effective through p53-dependent and independent pathways. For example, in lung cancer cell lines, it was found that MBZ treatment caused post-translational p53 stabilization and the downstream expression of p21 and MDM2 [14]. In p53-null lung cancer cells exposure to MBZ caused cytochrome $c$ accumulation, activation of caspase- 9 and caspase- 8 , and cleavage of PARP and procaspase- 3 . This independence of p53 status is also evident in the analysis of melanoma cells, where wild-type and mutant p53 cell lines were sensitive to MBZ [17].

There has been conflicting evidence regarding the effect that MBZ has on tumour neo-vascularisation, with some reports finding evidence that it has an anti-angiogenic effect and others finding none.

In the earliest work on the anti-cancer activity of MBZ, Mukhopadhyay and colleagues reported an anti-angiogenic effect on human lung cancer xenograft models [14]. However, in vivo analysis of adrenocortical cancer models failed to detect any anti-angiogenic activity compared to controls [16]. Some support for an anti-angiogenic action comes from an in silico study, which indicated that MBZ inhibits the action of VEGFR-2 by binding to it, a finding validated in vitro using a human umbilical vein endothelial cell (HUVEC) based angiogenesis functional assay [29]. Of note, the related drug albendazole has shown anti-angiogenic properties in an ovarian cancer model and in drug-resistant cell lines [30, 31], suggesting that an anti-angiogenic action may be common across a number of benzimidazoles.

To date, the effect of MBZ or other benzimidazole on the immune response in cancer has not been investigated, though there is some evidence that albendazole synergised to stimulate the cellular immune response in mice treated for alveolar echinococcosis with the immunotherapeutic agent liposomal muramyl tripeptide phosphatidylethanolamine (L-MTP-PE) used in the treatment of osteosarcoma [32]. There is also increasing evidence that existing microtubule disrupting agents used at low or metronomic doses, including the taxanes and vinca alkaloids, exert a positive immunomodulatory action that may help to reverse the immunosuppressive effect of cancer [33-36]. We can speculate, mechanistically, that some of this immunomodulatory action is related to microtubule dynamics. Therefore, there may be a similar effect with MBZ and other benzimidazoles, and this may also be a factor in the anti-cancer effects of these drugs.

\section{Our take}

\section{Next steps}

Based on the evidence summarised in Table 1, it is our contention that human clinical trials of mebendazole in a range of cancer types is warranted. The known pharmacokinetics, relatively low toxicity (even with extended high-dosing protocols), low cost, and strong pre-clinical evidence make this an ideal candidate for re-purposing. Currently, there are only two early phase clinical trials getting under way, both in glioma. In addition to these, the evidence suggests that candidate cancer types to take to human trial include:

- melanoma,

- non-small cell lung cancer,

- adrenocortical cancer, and

- colon cancer.

Additional cancer types, which should be further investigated in animal studies include:

- breast cancer,

- leukaemia, and

- osteosarcoma.

As with other anti-cancer agents, it is most likely that MBZ will be more effective in combination with other drugs or treatment modalities. It should be noted that the first two clinical trials are using MBZ with current standard of care treatment in glioma, which in this case means a combination protocol with other drugs, principally temozolomide. Given the primary putative mechanism of action—microtubule disruption-there are a number of additional agents that warrant investigation for synergy with MBZ, some of which are listed in the Appendix. 


\section{Other options}

Finally, improved efficacy may also be possible through improvements in the bioavailability of MBZ. As touched on previously, there is evidence that the combination of MBZ with cimetidine increases plasma levels of MBZ [12], potentially improving the therapeutic effect. An alternative means of increasing the bioavailability is through the liposomal encapsulation of MBZ. While this approach has not been explored in an oncological context, some work in this area have been done to enhance the anti-parasitic action of MBZ and other benzimidazole anti-helminthics, including one paper that explored the combined effect of a liposomal benzimidazole (albendazole) and cimetidine and reported a very significant increase in therapeutic effect (including a 75-94\% reduction in biomass of the hydatid cysts and a significant increase in survival time) in an animal model [37]. It is possible that a similar approach could yield improvements in the anti-cancer effect of MBZ.

\section{New protocols}

Adding MBZ to the existing standard of care protocols, as the first two clinical trials have done, provides an opportunity to test whether there are incremental improvements in outcomes compared to the standard of care alone. However, we should also seek opportunities to create new protocols that combine MBZ with other repurposed drugs with similar low toxicity and potential anti-cancer activity. The intention is to create novel treatment options that are multi-targeted and which present minimal risk of toxicity. Of necessity, given our current state of knowledge, the combinations proposed in the supplementary material are speculative and informed primarily by mechanistic considerations and pre-clinical data. Additional pre-clinical studies are required, but given the urgency of unmet patient needs and the low toxicity of the proposed combinations, it may be argued that small patient trials or even off-label usage may also be warranted.

\section{Conclusion}

The evidence for an anti-cancer effect of mebendazole treatment comes from in vitro, in vivo, in silico, and human data. Mechanistically, the microtubule action is well characterised in the laboratory and provides a similar rationale to some of the major classical chemotherapeutic drug classes, such as the taxanes and vinca alkaloids. With well-established pharmacokinetics and an excellent toxicity profile, this low-cost agent is a strong candidate for drug repurposing as an oncological treatment, both in combination with the existing standard treatments and alongside other candidate repurposing agents in a number of specific cancer types. We have outlined a number of these multi-drug combinations in the hope that clinicians can act upon this information to initiate clinical trials as a matter of some urgency.

\section{Acknowledgments}

Peter Nygren, Gregory Riggins and Gary Gallia.

\section{Author contributions}

Primary author: Pan Pantziarka. Contributing authors: Gauthier Bouche, Lydie Meheus, Vidula Sukhatme, Vikas P. Sukhatme. All authors read and approved the final manuscript.

\section{Competing interests}

The authors declare that they have no competing interests. All the authors are associated with not-for-profit organisations that aim to repurpose drugs for oncology treatments. 
Table 1. A summary of pre-clinical evidence by cancer type.

\begin{tabular}{|l|c|c|c|}
\hline \multicolumn{1}{|c|}{ Cancer Type } & In Vitro & In Vivo & Case Report/Trial \\
\hline Adrenocortical & {$[16]$} & {$[16]$} & {$[23]$} \\
\hline Breast & {$[14,21,20]$} & & {$[24]$} \\
\hline Colon & {$[14,20]$} & {$[14]$} & NCT01729260, NCT01837862 \\
\hline Glioma & {$[19]$} & {$[19]$} & \\
\hline Leukaemia & {$[20]$} & & \\
\hline Lung & {$[15]$} & {$[15]$} & \\
\hline Melanoma & {$[17,20]$} & {$[18]$} & \\
\hline Osteosarcoma & {$[14,22]$} & & \\
\hline Ovary & {$[14,20]$} & & \\
\hline
\end{tabular}

\section{References}

1. Ammann RW et al (1998) Long-term mebendazole therapy may be parasitocidal in alveolar echinococcosis $J$ Hepatol 29(6) 994-8 DOI: 10.1016/S0168-8278(98)80129-3

2. Rausch RL et al (1986) Consequences of continuous mebendazole therapy in alveolar hydatid disease-with a summary of a ten-year clinical trial Ann Trop Med Parasitol 80(4) 403-19

3. Janssen (2013) Vermox - Product monograph

4. Reuter S et al (2000) Benzimidazoles in the treatment of alveolar echinococcosis: a comparative study and review of the literature J Antimicrob Chemother 46(3) 451-6 DOI: 10.1093/jac/46.3.451 PMID: 10980173

5. Münst GJ, Karlaganis G and Bircher J (1980) Plasma concentrations of mebendazole during treatment of echinococcosis: preliminary results Eur J Clin Pharmacol 17(5) 375-8 DOI: 10.1007/BF00558451 PMID: $\underline{7418715}$

6. Braithwaite PA et al (1982) Clinical pharmacokinetics of high dose mebendazole in patients treated for cystic hydatid disease Eur J Clin Pharmacol 22(2) 161-9 DOI: 10.1007/BF00542462 PMID: 7094986

7. Dawson M and Watson TR (1985) The effect of dose form on the bioavailability of mebendazole in man Br J Clin Pharmacol 19(1) 87-90 DOI: 10.1111/j.1365-2125.1985.tb02617.x PMID: $\underline{3978024}$ PMCID: 1463790

8. Shcherbakov AM and Lur'e AA (1989) Clinical trial of the possibility of increasing the bioavailability of mebendazole using sunflower oil in echinococcosis Med Parazitol (Mosk) 3 46-9

9. Liu C et al (2012) Enhanced bioavailability and cysticidal effect of three mebendazole-oil preparations in mice infected with secondary cysts of Echinococcus granulosus Parasitol Res 111(3) 1205-11 DOI: 10.1007/s00436-012-2954-2 PMID: 22661241

10. García-Rodriguez JJ et al (2011) Changed crystallinity of mebendazole solid dispersion: improved anthelmintic activity Int J Pharm 403(1-2) 23-8 DOI: 10.1016/j.jjpharm.2010.10.002 
11. Chiba $Y$ et al (1991) Improvement of dissolution and bioavailability for mebendazole, an agent for human echinococcosis, by preparing solid dispersion with polyethylene glycol Cheml Pharm Bull 39(8) 2158-60 DOI: 10.1248/cpb.39.2158

12. Bekhti a and Pirotte J (1987) Cimetidine increases serum mebendazole concentrations. Implications for treatment of hepatic hydatid cysts Br J Clin Pharmacol 24(3) 390-2 DOI: 10.1111/j.1365-2125.1987.tb03186.x PMID: 3663452 PMCID: 1386263

13. Deva $S$ and Jameson M (2012) Histamine type 2 receptor antagonists as adjuvant treatment for resected colorectal cancer Cochrane Database Sys Rev 8(8) CD007814

14. Mukhopadhyay T et al (2002) Mebendazole elicits a potent antitumor effect on human cancer cell lines both in vitro and in vivo Clin Cancer Res 8(9) 2963-9 PMID: 12231542

15. Sasaki $\mathrm{J}$ et al (2002) The anthelmintic drug mebendazole induces mitotic arrest and apoptosis by depolymerizing tubulin in non-small cell lung cancer cells Mol Cancer Ther 1(13) 1201-9 PMID: 12479701

16. Martarelli $D$ et al (2008) Mebendazole inhibits growth of human adrenocortical carcinoma cell lines implanted in nude mice Cancer Chemother Pharmacol 61(5) 809-17 DOI: 10.1007/s00280-007-0538-0

17. Doudican $\mathrm{N}$ et al (2008) Mebendazole induces apoptosis via Bcl-2 inactivation in chemoresistant melanoma cells $\mathrm{Mol}$ Cancer Res 6(8) 1308-15 DOI: 10.1158/1541-7786.MCR-07-2159 PMID: 18667591

18. Doudican NA et al (2013) XIAP downregulation accompanies mebendazole growth inhibition in melanoma xenografts Anticancer Drugs 24(2) 181-8 DOI: 10.1097/CAD.0b013e32835a43f1

19. Bai $\mathrm{R}$ et al (2011) Antiparasitic mebendazole shows survival benefit in 2 preclinical models of glioblastoma multiforme Neuro Oncol 13(9) 974-82 DOI: 10.1093/neuonc/nor077 PMID: 21764822 PMCID: 3158014

20. Nygren $\mathrm{P}$ et al (2013) Repositioning of the anthelmintic drug mebendazole for the treatment for colon cancer $J$ Cancer Res Clin Oncol 139(12) 2133-40 DOI: 10.1007/s00432-013-1539-5 PMID: 24135855 PMCID: $\underline{3825534}$

21. Coyne CP, Jones $T$ and Bear R (2013) Gemcitabine-(C4-amide)-[anti-HER2/neu] anti-neoplastic cytotoxicty in dual combination with mebendazole against chemotherapeutic-resistant mammary adenocarcinoma J Clin Exp Oncol 02(02) DOI: 10.4172/2324$\underline{9110.1000109}$

22. Schmit JM (2013) In vitro anti-cancer effects of benzimidazoles on the canine osteosarcoma D17 cell line University of Illinois, Urbana, Illinois

23. Dobrosotskaya IY et al (2011) Mebendazole monotherapy and long-term disease control in metastatic adrenocortical carcinoma Endocr Prac 17(3) e59-62 DOI: 10.4158/EP10390.CR

24. Nygren $P$ and Larsson $R$ (2013) Drug repositioning from bench to bedside: Tumour remission by the antihelmintic drug mebendazole in refractory metastatic colon cancer Acta Oncol 57(3) 427-8

25. Laclette JP, Guerra G and Zetina C (1980) Inhibition of tubulin polymerization by mebendazole Biochem Biophys Res Commun 92(2) 417-23 DOI: 10.1016/0006-291X(80)90349-6 PMID: $\underline{7356473}$

26. Friedman PA and Platzer EG (1980) Interaction of anthelmintic benzimidazoles with Ascaris suum embryonic tubulin Biochim Biophys Acta 630(2) 271-8 DOI: 10.1016/0304-4165(80)90431-6 PMID: $\underline{7388055}$

27. Argyriou AA et al (2014) Chemotherapy-induced peripheral neuropathy in adults: a comprehensive update of the literature Cancer Manage Res 6 135-147 DOI: 10.2147/CMAR.S44261

28. Cata JP et al (2006) Clinical and experimental findings in humans and animals with chemotherapy-induced peripheral neuropathy Minerva Anestesiol 72(3) 151-69 PMID: 16493391 
29. Dakshanamurthy $S$ et al (2012) Predicting new indications for approved drugs using a proteochemometric method $J$ Med Chem 55(15) 6832-48 DOI: 10.1021/jm300576q PMID: 22780961 PMCID: $\underline{3419493}$

30. Pourgholami MH et al (2010) Potent inhibition of tumoral hypoxia-inducible factor 1alpha by albendazole BMC Cancer 10143 DOI: 10.1186/1471-2407-10-143 PMID: 20398289 PMCID: $\underline{2873385}$

31. Chu SWL et al (2009) Potent inhibition of tubulin polymerisation and proliferation of paclitaxel-resistant 1A9PTX22 human ovarian cancer cells by albendazole Anticancer Res 29(10) 3791-6 PMID: 19846910

32. Dvoroznáková E et al (2008) Imunomodulative effect of liposomized muramyltripeptide phosphatidylethanolamine (L-MTP-PE) on mice with alveolar echinococcosis and treated with albendazole Parasitol Res 103(4) 919-29 DOI: 10.1007/s00436-0081077-2 PMID: 18587669

33. Roselli $\mathrm{M}$ et al (2013) Effects of conventional therapeutic interventions on the number and function of regulatory $\mathrm{T}$ cells Oncoimmunol 2(10) e27025 DOI: $10.4161 /$ onci. 27025

34. Kaneno $\mathrm{R}$ et al (2009) Chemomodulation of human dendritic cell function by antineoplastic agents in low noncytotoxic concentrations J Trans/ Med 758 DOI: 10.1186/1479-5876-7-58 PMID: 19591684 PMCID: 2716306

35. Sevko A et al (2013) Antitumor effect of paclitaxel is mediated by inhibition of myeloid-derived suppressor cells and chronic inflammation in the spontaneous melanoma model J Immunol 190(5) 2464-71 DOI: 10.4049/jimmunol.1202781 PMID: 23359505 PMCID: $\underline{3578135}$

36. Sevko A et al (2012) Application of paclitaxel in low non-cytotoxic doses supports vaccination with melanoma antigens in normal mice J Immunotoxicol 9(3) 275-81 DOI: 10.3109/1547691X.2012.655343 PMID: 22449053

37. Wen $\mathrm{H}$ et al (1996) Pharmacology and efficacy of liposome-entrapped albendazole in experimental secondary alveolar echinococcosis and effect of co-administration with cimetidine Parasitology 113 (Pt 2) 111-21. DOI: 10.1017/S003118200006635X PMID: 8760312

\section{Appendix}

\section{Introduction}

The following drugs warrant further investigation in combination with mebendazole (MBZ), both in pre-clinical studies and potentially in clinical trials. These combinations listed in Table A1 have been selected on the basis of existing pre-clinical and clinical experience in each of the indications. In some cases, these combinations replicate existing protocols currently being tested in clinical trials, but substitute known and repurposed drugs for the newer and/or more toxic agents currently being investigated. All these proposed combinations are expected to display relatively low toxicity and use low cost and generally available agents.

\section{Higher-priority agents}

The agents listed below have a high degree of clinical evidence of efficacy and are currently either in clinical use in oncology or are currently being investigated in clinical trials. They have been selected as potential agents to be used in combination with MBZ. Note that these drugs are not listed in order of priority.

- Metformin: There is pre-clinical evidence that metformin potentiates the action of existing microtubule disrupting drugs in a range of cancer types, including endometrial cancers and paediatric sarcomas [1-3]. Given the low toxicity of metformin and its potential as an anti-cancer agent, the combination with MBZ should be explored, both in animal models and potentially in small clinical trials. 
- Metronomic chemotherapy: While there is intense interest in the area of metronomic chemotherapy using taxanes or vinca alkaloids, progress has been restricted because of a lack of oral formulations of many of these drugs, with the exception of oral vinorelbine. Where existing microtubule targeting drugs without oral formulations are used in metronomic settings, it is normally as a weekly infusion in combination with daily dosing of oral cyclophosphamide or capecitabine. A number of clinical trials using oral vinorelbine have reported both low toxicity and evidence of clinical benefit in advanced cancers [4, 5]. MBZ also offers the possibility of exploring daily oral dosing of a microtubule disrupting agent in combination with low dose oral chemotherapy drugs and other agents used in such protocols (e.g. celecoxib or other anti-inflammatory). It is theorised that one of the principal methods of action of metronomic chemotherapy is through inhibition of neo-angiogenesis, and that escape from angiogenic control is associated with treatment failure. The addition of MBZ with existing metronomic protocols may increase the anti-angiogenic effect of treatment and prolong the therapeutic benefit.

- Taxanes or Vinca Alkaloids: Combinations of microtubule targeting agents, for example, paclitaxel or docetaxel and vinorelbine, act synergistically, and there are numerous trials exploring multiple combinations of different microtubule agents [6]. Pre-clinical evidence shows that the benzimidazole flubendazole synergises with vincristine and vinblastine in vitro and in vivo [7]. Given that MBZ has such low toxicity in comparison to many existing microtubule agents, combination therapy of MBZ with taxanes or vinca alkaloid drugs would seem a promising avenue to explore in human trials. One prospect is to combine MBZ with oral vinorelbine, offering the prospect of dual oral microtubule disrupting drugs, with low toxicity, either in standard dosing of vinorelbine or at metronomic dosing of both agents.

- Albendazole or other benzimidazole: There is evidence that the different benzimidazoles vary in their molecular targets and that combining them may improve efficacy and reduce the risks of acquired resistance. While this approach has not been explored in a cancer setting, there is pre-clinical and clinical evidence that the combination of MBZ and albendazole is a more effective treatment in certain hard to treat parasitic conditions [8, 9]. There is also some in vitro and in vivo evidence where albendazole exerts an anti-angiogenic action by down-regulating vascular endothelial growth factor (VEGF), an effect mediated through inhibition of tumoural hypoxia inducible factor (HIF-1 $\alpha$ ) [10]. As HIF-1 $\alpha$ is implicated in multi-drug resistance in cancer, the combination of MBZ and albendazole warrants further investigation in drug-resistant tumours.

- Itraconazole: The anti-fungal drug itraconazole has shown some evidence of having anti-cancer activity, possibly through an anti-angiogenic action and inhibition of Hedgehog signalling pathway [11, 12]. It is currently being investigated in a number of clinical trials, principally in metastatic prostate cancer (e.g. www.clinicaltrials.gov/ct2/show/NCT00887458). A recently completed Phase II trial in basal cell carcinoma showed that in eight previously untreated patients with multiple tumours, four showed partial response and four had stable disease. In contrast, patients previously treated with vismodegib showed no significant changes in proliferation or tumour size [13]. There is laboratory evidence that Hedgehog inhibition can reverse resistance to taxane chemotherapy in a range of cell lines, including ovarian [14] and prostatic cancer [15]. There is specific evidence that itraconazole itself is able to reverse multi-drug resistance in resistant HeLa cells, at least in vitro, at doses achievable in humans [16].

- Cimetidine: The $\mathrm{H} 2$ receptor antagonist cimetidine, primarily used to treat peptic ulcers and heartburn, has shown in vitro and in vivo anticancer activity in a range of cell types and animal models, with a number of possible methods of action [17], including favourable impact on the immune system. A recent Cochrane Review suggested that there was a trend towards improved survival outcomes when cimetidine is used as perioperative and/or adjuvant treatment for early-stage colorectal cancer [18]. As was mentioned previously, there is also evidence that cimetidine can increase the plasma levels of MBZ [19]. A combination of cimetidine and MBZ would therefore be of interest, particularly in colorectal cancer where there is human evidence that each agent has some anticancer activity.

- Diclofenac: The non-steroidal anti-inflammatory drug (NSAID) is a commonly used anti-inflammatory analgesic with known activity as a COX-2 inhibitor, and is available both in topical and oral form. While there is evidence that perioperative or intraoperative diclofenac may be associated with lower risk of cancer recurrence or metastases following surgery [20], there are also active investigations of its direct anticancer role. In particular, diclofenac has shown pre-clinical activity in a range of cancer types, with in vivo evidence in melanoma [21] and ovarian cancer [22] in particular. Clinically, diclofenac has been used in the treatment of recurrent or aggressive desmoid tumours, including cases where diclofenac was combined with the microtubule targeting drug vinblastine [23]. Celecoxib, another COX-2 inhibitor, has also been used in combination with metronomically dosed paclitaxel in metastatic melanoma and shown some evidence of clinical activity [24]. Given the pre-clinical evidence of MBZ activity against melanoma and ovarian cancer and the similar evidence for diclofenac, early phase human trials of a combination treatment would be warranted. 
- Chloroquine/Hydroxychloroquine: The anti-malarial drugs chloroquine and hydroxychloroquine are under active investigation in a range of clinical trials for cancer in combination with radiotherapy and/or alongside existing chemotherapy regimens. The putative mechanism of action of chloroquine is that it acts as an inhibitor of autophagy, acting therefore to restrict the ability of cancer cells to move to an autophagic state such that they move into apoptosis in response to cellular stresses initiated by chemotherapy or radiotherapy [25]. A particular focus of much pre-clinical and clinical research with chloroquine and hydroxychloroquine is in glioblastoma, with initial results from one small clinical trial showing a tendency to longer overall survival, though the result of the small sample size is not statistically significant [26]. The rationale for a combination of MBZ and chloroquine/hydroxychloroquine is stronger for those indications where the level of evidence for each agent is stronger, that is in glioblastoma and melanoma, and therefore human trials in these cancer types are warranted.

- Clarithromycin: A well-established macrolide antibiotic, clarithromycin has been used in an oncological setting for the eradication of Helicobacter Pylori infection or as a treatment for treatment-associated mycobacterial infection. It has also been used in combination therapy with lenalidomide, and dexamethasone for the treatment of multiple myeloma [27] or as a monotherapy for B cell lymphoma [28]. One mechanism of action that is being actively explored in relation to the anticancer effects of clarithromycin is a suggested action as an inhibitor of autophagy [29]. The role of autophagy in cancer is a complex one, but there is evidence that the inhibition of autophagy in established tumours abrogates a key survival mechanism utilised by cancer cells to protect themselves from a range of cancer treatments, including chemotherapy, radiotherapy and targeted therapies [30]. There are a number of clinical trials combining the autophagy inhibitor chloroquine with a range of chemotherapeutic drugs, including microtubule disrupters, in different cancers. The combination of MBZ and clarithromycin would mirror the underlying strategy of some of these existing trials but using low-cost and low-toxicity agents. This would be an approach most warranted in gliomas, where it has been shown that the inhibition of autophagy with chloroquine in addition to standard treatment improved survival [26].

\section{Other agents}

The drugs listed below may also be suitable for combination treatments with MBZ and other agents, however, the evidence is not as strong and therefore this list must be viewed as more speculative.

- 2-Methoxyestradiol (2Me): A natural metabolite of estradiol, $2 \mathrm{Me}$ has shown promising anti-cancer activity in a number of clinical trials and is currently being developed as a drug under the trade name of Panzem (EntreMed Inc), with trials on-going in a range of solid tumours. Proposed methods of action include anti-angiogenesis, suppression of microtubule dynamics and inhibition of proliferation [31]. There have been a number of in vitro and in vivo studies assessing the synergistic action of $2 \mathrm{Me}$ with other microtubule-targeting drugs, including a recent study that assessed the combination of $2 \mathrm{Me}$ and albendazole in a xenograft colorectal cancer model and reported a significantly improved survival time [32].

- Losartan: The angiotensin II receptor antagonist losartan, used mainly to treat hypertension, is currently being investigated as a possible anticancer therapeutic, primarily for its role in counter-acting the reduced vascular perfusion caused by physical stresses within the tumour mass [33]. The direct role of angiotensin II in cancer progression, particularly with regards to the up-regulation of angiogenesis is also being investigated, and therefore losartan may also have an anticancer effect through its primary function as an angiotensin II receptor blocker (ARB) [34]. Tumour hypoxia and lack of vascular perfusion are often causes of treatment failure in chemotherapy, and therefore a possibility also with MBZ treatment. Therefore, a combination treatment of losartan and MBZ, particularly in solid tumours, such as osteosarcoma, would be of interest.

- Omega 3 PUFAs: There is pre-clinical evidence that omega 3 fatty acids eicosapentaenoic acid (EPA) and docosahexaenoic acid (DHA) can have chemosensitising effects in a range of cancer cell types and for a range of standard chemotherapeutic drugs [35]. There is also limited clinical evidence that oral supplementation with EPA/DHA can improve outcomes in colorectal and advanced non-small cell lung cancer [36, 37]. Given this sensitisation of tumours to chemotherapies, and the known improvement in MBZ bioavailability when taken with a fatty meal [38], the combination of EPA/DHA and MBZ should also be explored. 
Table A1. Proposed drug combinations with MBZ for specific indications.

\begin{tabular}{|c|c|c|}
\hline Disease & Targets & Drug Combination \\
\hline Malignant Melanoma & $\begin{array}{l}\text { Microtubule disruption, inhibition of } \\
\text { autophagy, anti-angiogenic and } \\
\text { immunomodulation }\end{array}$ & $\begin{array}{l}\text { Hydroxychloroquine (NCT00962845) } \\
\text { Diclofenac or Celecoxib [24] } \\
\text { Oral cyclophosphamide [39] }\end{array}$ \\
\hline NSCLC & $\begin{array}{l}\text { Microtubule disruption, AMPK/mTOR, } \\
\text { Hedgehog signalling, COX-2 inhibition }\end{array}$ & $\begin{array}{l}\text { Metformin (NCT01997775) } \\
\text { Itraconazole [40] } \\
\text { Diclofenac or Celecoxib (NCTO0520845) }\end{array}$ \\
\hline Adrenocortical Carcinoma & $\begin{array}{l}\text { Microtubule disruption, anti-angiogenic, } \\
\text { Hedgehog signalling }\end{array}$ & $\begin{array}{l}\text { Itraconazole } \\
\text { Oral cyclophosphamide [41] }\end{array}$ \\
\hline Glioblastoma Multiforme & $\begin{array}{l}\text { Inhibition of autophagy, microtubule } \\
\text { disruption, Hedgehog pathway inhibition, } \\
\text { anti-angiogenic }\end{array}$ & $\begin{array}{l}\text { Hydroxychloroquine (NCT00224978) } \\
\text { Itraconazole }\end{array}$ \\
\hline Colorectal Carcinoma & $\begin{array}{l}\text { Microtubule disruption, AMPK/mTOR, } \\
\text { immunomodulation, anti-histamine, COX-2 }\end{array}$ & $\begin{array}{l}\text { Metformin (NCT01941953) } \\
\text { Cimetidine [42] } \\
\text { Diclofenac } \\
\text { Oral vinorelbine [43] }\end{array}$ \\
\hline $\begin{array}{l}\text { Osteosarcoma/ Soft-tissue } \\
\text { Sarcoma }\end{array}$ & $\begin{array}{l}\text { Microtubule disruption, AMPK/mTOR, IGF-I, } \\
\text { Hedgehog pathway inhibition, tumour } \\
\text { vascularity, anti-angiogenic }\end{array}$ & $\begin{array}{l}\text { Metformin } \\
\text { Itraconazole } \\
\text { Losartan } \\
\text { Oral cyclophosphamide [44] }\end{array}$ \\
\hline Acute Myeloid Leukaemia & $\begin{array}{l}\text { Microtubule disruption, induction of apop- } \\
\text { tosis }\end{array}$ & $\begin{array}{l}\text { Albendazole or oral vinorelbine [45] } \\
\text { Diclofenac }\end{array}$ \\
\hline $\begin{array}{l}\text { Breast Cancer (ER+ invasive } \\
\text { ductal carcinoma) }\end{array}$ & $\begin{array}{l}\text { Microtubule disruption, AMPK/mTOR, } \\
\text { anti-angiogenic }\end{array}$ & $\begin{array}{l}\text { Metformin (NCT01929811) } \\
\text { Oral cyclophosphamide and/or oral } \\
\text { vinorelbine (NCT00954135) }\end{array}$ \\
\hline Ovarian Carcinoma & Ovarian Carcinoma & $\begin{array}{l}\text { Metformin (NCT02050009) } \\
\text { Itraconazole } \\
\text { Diclofenac (NCT01124435) }\end{array}$ \\
\hline
\end{tabular}

Note that references to clinical trials or published papers are indicative of trials or case reports where the drug (or analogue) has been used for the specific indication.

\section{References}

1. Iliopoulos D, Hirsch HA and Struhl K (2011) Metformin decreases the dose of chemotherapy for prolonging tumor remission in mouse xenografts involving multiple cancer cell types Cancer Res (9) 3196-201 DOI: 10.1158/0008-5472.CAN-10-3471 PMID: 21415163 PMCID: $\underline{3085572}$

2. Hanna RK et al (2012) Metformin potentiates the effects of paclitaxel in endometrial cancer cells through inhibition of cell proliferation and modulation of the mTOR pathway Gynecol Oncol 125(2) 458-69 DOI: 10.1016/j.ygyno.2012.01.009 PMID: 22252099 PMCID: $\underline{3322276}$

3. Garofalo C et al (2013) Metformin as an adjuvant drug against pediatric sarcomas: Hypoxia limits therapeutic effects of the drug PloS One 8(12) e83832 DOI: 10.1371/journal.pone.0083832 
4. Briasoulis $\mathrm{E}$ et al (2013) Dose selection trial of metronomic oral vinorelbine monotherapy in patients with metastatic cancer: a hellenic cooperative oncology group clinical translational study BMC Cancer 13(1) 263 DOI: 10.1186/1471-2407-13-263 PMID: 23718900 PMCID: $\underline{3674943}$

5. Cazzaniga ME et al (2014) Efficacy and safety of the all-oral schedule of metronomic vinorelbine and capecitabine in locally advanced or metastatic breast cancer patients: The phase I-II VICTOR-1 study Int J Breast Cancer 2014769790 DOI: 10.1155/2014/769790 PMID: 24551455 PMCID: $\underline{3914392}$

6. Jordan MA and Wilson L (2004) Microtubules as a target for anticancer drugs Nat Rev Cancer 4(4) 253-65 DOI: $\underline{10.1038 / n r c 1317}$ PMID: 15057285

7. Spagnuolo PA et al (2010) The antihelmintic flubendazole inhibits microtubule function through a mechanism distinct from Vinca alkaloids and displays preclinical activity in leukemia and myeloma Blood 115(23) 4824-33 DOI: 10.1182/blood-2009-09243055 PMID: 20348394

8. Keiser J et al (2012) Effect of combinations of marketed human anthelmintic drugs against Trichuris muris in vitro and in vivo Parasit Vectors 5(1) 292 DOI: $\underline{10.1186 / 1756-3305-5-292}$ PMID: 23231753 PMCID: $\underline{3533970}$

9. Namwanje H, Kabatereine NB and Olsen A (2011) Efficacy of single and double doses of albendazole and mebendazole alone and in combination in the treatment of Trichuris trichiura in school-age children in Uganda Tran R Soc Trop Med Hyg 105 (10) 586-590. DOI: 10.1016/j.trstmh.2011.07.009

10. Pourgholami MH et al (2010) Potent inhibition of tumoral hypoxia-inducible factor 1alpha by albendazole BMC Cancer 10143 DOI: 10.1186/1471-2407-10-143 PMID: 20398289 PMCID: $\underline{2873385}$

11. Chong CR et al (2007) Inhibition of angiogenesis by the antifungal drug itraconazole ACS Chem Biol 2(4) 263-70 DOI: $10.1021 /$ cb600362d PMID: 17432820

12. Antonarakis ES et al (2013) Repurposing itraconazole as a treatment for advanced prostate cancer: a noncomparative randomized phase II trial in men with metastatic castration-resistant prostate cancer Oncologist 18(2) 163-73 DOI: 10.1634/ theoncologist.2012-314 PMID: 23340005 PMCID: 3579600

13. Kim DJ et al (2014) Open-label, exploratory Phase II trial of oral itraconazole for the treatment of basal cell carcinoma 32(8) J Clin Oncol 1-7 DOI: 10.1200/JCO.2013.49.9525

14. Steg AD et al (2012) Smoothened antagonists reverse taxane resistance in ovarian cancer Mol Cancer Therap 11(7) 1587-97 DOI: $10.1158 / 1535-7163 . M C T-11-1058$

15. Singh $\mathrm{S}$ et al (2012) Chemoresistance in prostate cancer cells is regulated by miRNAs and Hedgehog pathway PloS One 7(6) e40021 DOI: 10.1371/journal.pone.0040021 PMID: 22768203 PMCID: $\underline{3386918}$

16. lida $\mathrm{N}$ et al (2001) Reversal effects of antifungal drugs on multidrug resistance in MDR1-overexpressing HeLa cells Biol Pharm Bull 24(9) 1032-6 DOI: $10.1248 / \mathrm{bpb} .24 .1032$ PMID: 11558564

17. Kubecova M et al (2011) Cimetidine: an anticancer drug? Eur J Pharm Sci 42(5) 439-44 DOI: 10.1016/j.ejps.2011.02.004 PMID: $\underline{21329756}$

18. Deva $S$ and Jameson M (2012) Histamine type 2 receptor antagonists as adjuvant treatment for resected colorectal cancer Cochrane Database Syst Rev 8(8) CD007814 PMID: 22895966

19. Bekhti A and Pirotte J (1987) Cimetidine increases serum mebendazole concentrations. Implications for treatment of hepatic hydatid cysts Br J Clin Pharmacol 24(3) 390-2 DOI: 10.1111/j.1365-2125.1987.tb03186.x PMID: 3663452 PMCID: 1386263

20. Forget $P$ et al (2014) Intraoperative use of ketorolac or diclofenac is associated with improved disease-free survival and overall survival in conservative breast cancer surgery $\mathrm{Br} J$ Anaesth 1-6 
21. Gottfried E et al (2013) New aspects of an old drug--diclofenac targets MYC and glucose metabolism in tumor cells PloS One 8(7) e66987 DOI: $\underline{10.1371 / j o u r n a l . p o n e .0066987 ~ P M I D: ~} \underline{23874405}$ PMCID: $\underline{3706586}$

22. Zerbini LF et al (2011) Combinatorial effect of non-steroidal anti-inflammatory drugs and NF-kB inhibitors in ovarian cancer therapy PloS One 6(9) e24285 DOI: 10.1371/journal.pone.0024285

23. Lackner $\mathrm{H}$ et al (2004) Multimodal treatment of children with unresectable or recurrent desmoid tumors: an 11-year longitudinal observational study $J$ Pediatr Hematol Oncol 26(8) 518-22 DOI: 10.1097/01.mph.0000130219.26284.b3 PMID: 15284591

24. Bhatt RS et al (2010) A phase 2 pilot trial of low-dose, continuous infusion, or "metronomic" paclitaxel and oral celecoxib in patients with metastatic melanoma Cancer 116(7) 1751-6 DOI: 10.1002/cncr.24902 PMID: 20120033 PMCID: 2847062

25. Carew JS, Kelly KR and Nawrocki ST (2012) Autophagy as a target for cancer therapy: new developments Cancer Manag Res 4 357-65 PMID: 23091399 PMCID: $\underline{3474143}$

26. Sotelo J, Briceño E and López-González MA (2006) Adding chloroquine to conventional treatment for glioblastoma multiforme: a randomized, double-blind, placebo-controlled trial Ann Intern Med 144(5) 337-43 DOI: 10.7326/0003-4819-144-5-20060307000008 PMID: $\underline{16520474}$

27. Niesvizky R et al (2008) BiRD (Biaxin [clarithromycin]/Revlimid [lenalidomide]/dexamethasone) combination therapy results in high complete- and overall-response rates in treatment-naive symptomatic multiple myeloma Blood 111(3) 1101-9 DOI: 10.1182/blood-2007-05-090258

28. Govi S et al (2010) Six-month oral clarithromycin regimen is safe and active in extranodal marginal zone B-cell lymphomas: final results of a single-centre phase II trial Br J Haematol 150(2) 226-9 PMID: 20433679

29. Moriya $S$ et al (2013) Macrolide antibiotics block autophagy flux and sensitize to bortezomib via endoplasmic reticulum stress-mediated CHOP induction in myeloma cells Int J Oncol 42(5) 1541-50 PMID: 23546223 PMCID: 3661227

30. Maes H et al (2013) Autophagy: shaping the tumor microenvironment and therapeutic response Trends Mol Med 19(7) 428-46 DOI: 10.1016/j.molmed.2013.04.005 PMID: 23714574

31. Sweeney C et al (2005) A phase II multicenter, randomized, double-blind, safety trial assessing the pharmacokinetics, pharmacodynamics, and efficacy of oral capsules in hormone-refractory prostate cancer Clin Cancer Res 11(18) 6625-33 DOI: 10.1158/1078-0432.CCR-05-0440 PMID: 16166441

32. Ehteda $\mathrm{A}$ et al (2013) Combination of albendazole and 2-methoxyestradiol significantly improves the survival of HCT-116 tumor-bearing nude mice BMC Cancer 13(1) 86 DOI: 10.1186/1471-2407-13-86 PMID: 23432760 PMCID: $\underline{3606618}$

33. Chauhan VP et al (2013) Angiotensin inhibition enhances drug delivery and potentiates chemotherapy by decompressing tumour blood vessels Nat Commun 42516 DOI: 10.1038/ncomms3516 PMID: 24084631 PMCID: $\underline{3806395}$

34. Chen X et al (2013) Angiotensin II type 1 receptor antagonists inhibit cell proliferation and angiogenesis in breast cancer Cancer Lett 328(2) 318-24 DOI: 10.1016/j.canlet.2012.10.006

35. Hajjaji $N$ and Bougnoux $P$ (2013) Selective sensitization of tumors to chemotherapy by marine-derived lipids: a review Cancer Treat Rev 39(5) 473-88 DOI: 10.1016/j.ctrv.2012.07.001

36. Cockbain AJ et al (2014) Anticolorectal cancer activity of the omega-3 polyunsaturated fatty acid eicosapentaenoic acid Gut DOI: $10.1136 /$ gutjinl-2013-306445 PMID: 24470281

37. Murphy RA et al (2011) Supplementation with fish oil increases first-line chemotherapy efficacy in patients with advanced nonsmall cell lung cancer Cancer 117(16) 3774-80 DOI: 10.1002/cncr.25933 PMID: 21328326

38. Janssen (2013) Vermox - Product monograph 
39. Borne E et al (2010) Oral metronomic cyclophosphamide in elderly with metastatic melanoma Invest New Drugs 28(5) 684-9

40. Rudin CM et al (2013) Phase 2 study of pemetrexed and itraconazole as second-line therapy for metastatic nonsquamous non-small-cell lung cancer $J$ Thorac Oncol 8(5) 619-23

41. Ferrero A et al (2013) Metronomic chemotherapy may be active in heavily pre-treated patients with metastatic adreno-cortical carcinoma J Endocrinol Invest 36(3) 148-52

42. Matsumoto $S$ et al (2002) Cimetidine increases survival of colorectal cancer patients with high levels of sialyl Lewis- $X$ and sialyl Lewis-A epitope expression on tumour cells Br J Cancer 86(2) 161-7

43. Briasoulis E et al (2009) Dose-ranging study of metronomic oral vinorelbine in patients with advanced refractory cancer Clin Cancer Res 15(20) 6454-61

44. André $\mathrm{N}$ et al (2011) Pilot study of a pediatric metronomic 4-drug regimen Oncotarget 2(12) 960-5

45. Shukla N et al (2014) Phase II trial of clofarabine with topotecan, vinorelbine, and thiotepa in pediatric patients with relapsed or refractory acute leukemia Pediatr Blood Cancer 61(3) 431-5 